\title{
Comparison of solar radio and extreme ultraviolet synoptic limb charts during the present solar maximum
}

\author{
A. J. Oliveira e Silva ${ }^{1}$, C. L. Selhorst ${ }^{1,2}$, P. J. A. Simões ${ }^{3}$, and C. G. Giménez de Castro ${ }^{4,5}$ \\ ${ }^{1}$ IP\&D - Universidade do Vale do Paraíba - UNIVAP, São José dos Campos, SP, Brazil \\ e-mail: caiuslucius@gmail.com \\ 2 NAT - Núcleo de Astrofísica Teórica, Universidade Cruzeiro do Sul, São Paulo, SP, Brazil \\ 3 SUPA School of Physics and Astronomy, University of Glasgow, G12 8QQ, UK \\ ${ }^{4}$ CRAAM, Universidade Presbiteriana Mackenzie, São Paulo, Brazil \\ 5 IAFE, Universidad de Buenos Aires/CONICET, Buenos Aires, Argentina
}

Received 19 November 2015 / Accepted 10 June 2016

\begin{abstract}
Aims. The present solar cycle is particular in many aspects: it had a delayed rising phase, it is the weakest of the last $100 \mathrm{yrs}$, and it presents two peaks separated by more than one year. To understand the impact of these characteristics on the solar chromosphere and coronal dynamics, images from a wide wavelength range are needed. In this work we use the $17 \mathrm{GHz}$ radio continuum, which is formed in the upper chromosphere and the extreme ultraviolet (EUV) lines 304 and $171 \AA$, that come from the transition region (He II, $T \sim 6-8 \times 10^{4} \mathrm{~K}$ ) and the corona (Fe IX, X, $T \sim 10^{6} \mathrm{~K}$ ), respectively. We extend upon a previous similar analysis, and compare the mean equatorial and polar brightening behavior at radio and EUV wavelengths during the maximum of the present solar cycle, covering the period between 2010 and 2015 .

Methods. We analyze daily images at 304 and $171 \AA$ obtained by the Atmospheric Imaging Assembly (AIA) on board the Solar Dynamics Observatory (SDO). The $17 \mathrm{GHz}$ maps were obtained by the Nobeyama Radioheliograph (NoRH). To construct synoptic limb charts, we calculated the mean emission of delimited limb areas with $100^{\prime \prime}$ wide and angular separation of $5^{\circ}$.

Results. At the equatorial region, the results show a hemispheric asymmetry of the solar activity. The northern hemisphere dominance is coincident with the first sunspot number peak, whereas the second peak occurs concurrently with the increase in the activity at the south. The polar emission reflects the presence of coronal holes at both EUV wavelengths, moreover, the $17 \mathrm{GHz}$ polar brightenings can be associated with the coronal holes. Until 2013, both EUV coronal holes and radio polar brightenings were more predominant at the south pole. Since then they have not been apparent in the north, but thus appear in the beginning of 2015 in the south as observed in the synoptic charts.

Conclusions. This work strengthens the association between coronal holes and the $17 \mathrm{GHz}$ polar brightenings as it is evident in the synoptic limb charts in agreement with previous case study papers. The enhancement of the radio brightness in coronal holes is explained by the presence of bright patches closely associated with the presence of intense unipolar magnetic fields. However, observations with better spatial resolution and also at different radio wavelengths will be necessary to fully understand the physical mechanisms that link these features.
\end{abstract}

Key words. Sun: general - Sun: radio radiation - Sun: UV radiation - Sun: transition region - Sun: corona

\section{Introduction}

Coronal holes are low density regions caused by the presence of locally open magnetic field lines, which are often observed at the solar poles. Owing to the low density they are observed as dark regions in coronal extreme (EUV) lines and X-rays. This characteristic is also observed at low radio frequencies formed in the corona, however, at frequencies higher than $15 \mathrm{GHz}$ bright regions inside the the coronal holes are often observed (see for example: Gopalswamy et al. 1999; Brajša et al. 2007).

The presence of bright polar regions is clear in $17 \mathrm{GHz}$ maps, obtained by the Nobeyama Radioheliograph (NoRH, Nakajima et al. 1994), and shows a cycle mimicking the polar faculae cycle (Selhorst et al. 2011; Gopalswamy et al. 2012; Nitta et al. 2014). Selhorst et al. (2010) proposed that these bright patches could be generated by small scale magnetic regions around the positions of intense magnetic patches $(\mathrm{kG})$, that coincide with the polar faculae (Tsuneta et al. 2008). The presence of $17 \mathrm{GHz}$ bright patches were also observed at lower latitudes and have been associated with the enhanced unipolar magnetic regions underlying the coronal holes (Gopalswamy et al. 1999; Maksimov et al. 2006). On the contrary, small bright structures observed in EUV filter images do not have a one-to-one correspondence with the bright $17 \mathrm{GHz}$ patches (Nindos et al. 1999; Riehokainen et al. 2001; Nitta et al. 2014); for this reason, Nitta et al. (2014) suggest that most of the small bright structures are artifacts created by the image synthesis and deconvolution used in NoRH maps.

Selhorst et al. (2010) compared the variation of the polar mean emission at radio $(17 \mathrm{GHz})$ and EUV (304 and $171 \AA$ ), obtained with the Extreme ultraviolet Imager Telescope (EIT) on board the Solar and Heliospheric Observatory (SOHO). The 304 and $171 \AA$ A polar brightenings have distinct behaviors during the solar cycle. While $171 \AA$ is correlated with the solar cycle, $304 \AA$ is anticorrelated and in agreement with $17 \mathrm{GHz}$. periods. The authors addressed the $171 \AA$ variations to the presence of coronal holes, whereas the $304 \AA$ behavior was attributed to 
polar structures in the lower atmosphere, which also determine the polar emission at the $17 \mathrm{GHz}$ radio continuum.

The formation of the He II $304 \AA$ emission depends on the conditions of the lower atmosphere (transition region and chromosphere), but can also be affected by direct photoionization produced by coronal EUV photons (Hirayama 1971; Zirin 1975; Jordan 1975; Andretta \& Jones 1997). Worden et al. (1999) compared the He II $304 \AA$ emission from different atmospheric structures, namely plage, enhanced network, active networks, and quiet chromosphere, using EIT/SOHO images, over a period of two years during the solar minimum. They found that the intensity contrast of those structures (averaged over their areas) remains constant with time, when compared to the quiet chromosphere. The quiet chromosphere emission also does not vary during the period, however, their definition of quiet chromosphere also includes coronal holes. Worden et al. also suggest that because of the constancy of the quiet chromosphere irradiance in the analyzed period, the He II $304 \AA$ long-term variations could be traced to the magnetic activity. This idea is supported by Pauluhn \& Solanki (2003), who investigated magnetograms and chromospheric EUV irradiance during the period 1996-2000. They found that in quiet regions of the solar disk there was an increase of 10 to $20 \%$ in the magnetic flux density, and a corresponding increase in irradiance in He I $584 \AA$, with similar characteristics to He II $304 \AA$ emission in the different atmospheric structures (e.g. coronal holes, network regions Glackin et al. 1978). Glackin et al. (1978) indicated that both He I $584 \AA$ and He II $304 \AA$ show limb brightening in quiet Sun regions and limb darkening in coronal holes. Finally, Del Zanna \& Andretta (2011) showed that both He I $584 \AA$ and He II $304 \AA$ are correlated well with the 10.7 cm flux over the period 1998-2010.

In this paper, we analyze the relationship between the limb emission at radio $(17 \mathrm{GHz})$ and the EUV lines (304 and $171 \AA$ ) using synoptic limb charts covering the period between 2010 and 2015. The EUV images were taken by the Atmospheric Imaging Assembly (AIA; Lemen et al. 2012) aboard the Solar Dynamics Observatory (SDO), launched in 2010, whereas the $17 \mathrm{GHz}$ maps were obtained by the Nobeyama Radioheliograph. These data are described in the following section, with the results presented in Sect. 3. The discussion and main conclusions are presented in Sect. 4.

\section{Data analysis}

For the analysis presented here we use daily AIA EUV images taken with narrowband filters centered on He II $304 \AA$ and Fe IX $171 \AA$. While the $304 \AA$ line is an emission line formed in the transition region (TR), with temperatures ranging between 6 and $8 \times 10^{4} \mathrm{~K}$, the $171 \AA$ comes from coronal heights, with temperatures of $T \sim 10^{6} \mathrm{~K}$. Images are $1024 \times 1024$ pixel size, and present an angular resolution of around $2 " .40$. The prereduced images were obtained at the Joint Science Operations Center ${ }^{1}$. To read the images, the standard AIA routines were used (Boerner et al. 2012), which are part of the Solar SoftWare (SSW) package (Freeland \& Handy 1998). While the $304 \AA$ images presented a constant exposure time of $2.90 \mathrm{~s}$, at $171 \AA$ the first 68 days of the mission used exposure time of $2.90 \mathrm{~s}$ and $2.00 \mathrm{~s}$ after that. To make the 171 Ådata base uniform, the digital number (DN) of all maps were normalized to exposure time of $2.00 \mathrm{~s}$.

1 http://jsoc.stanford.edu/data/aia/synoptic/
At $17 \mathrm{GHz}(1.76 \mathrm{~cm})$, solar maps have been obtained daily by the NoRH interferometer since 1992 with the same quality resolution $\left(10-18^{\prime \prime}\right)$. We analyzed the online daily presynthesized images ${ }^{2}$, which are taken during local noon using a $10 \mathrm{~s}$ integration time. These maps use the Steer CLEAN algorithm (Steer et al. 1984), which has the advantage of restoring diffuse features with a ratio better than 1:300 between the rms noise level and the peak brightness in the images (Koshiishi 2003), which is very good for our work. The $17 \mathrm{GHz}$ maps have $512 \times 512$ pixels and a spatial resolution of 4.91 arcsecs. Emission at $17 \mathrm{GHz}$ mostly comes from the chromosphere (opacity $\tau=1$ ), where the local temperature and electron density are estimated to be $\sim 9.6 \times 10^{3} \mathrm{~K}$ and $\sim 9.3 \times 10^{9} \mathrm{~cm}^{-3}$, respectively (Selhorst et al. 2005).

To investigate the relationship between the limb emission at radio and EUV wavelengths, we chose to construct synoptic limb charts. To produce these charts, we calculated the mean emission of delimited limb areas $100^{\prime \prime}$ wide and angular separation of $5^{\circ}$. Figure 1 shows examples of solar images obtained on May 14, 2010, at the three wavelengths studied here at $17 \mathrm{GHz}, 304 \AA$ and $171 \AA$, respectively, with the delimited limb region used to construct the synoptic limb maps. The angular positions were defined clockwise with $0^{\circ}$ at the west $\operatorname{limb}, 90^{\circ}$ at the south pole, $180^{\circ}$ at the east and $270^{\circ}$ at the north pole.

This procedure results in mean intensity profiles along the solar limb. To compare the variation of these profiles across the years, we normalized the maps by the quiet Sun temperature (or data number (DN) for AIA images). The mean relative intensity $\left(I_{R}\right)$ profiles for each map are shown in the bottom panels of Fig. 1. Distinct structures can be observed at the poles in the images:

i) The south pole presents a coronal hole at $171 \AA$ (Fig. 1c), which results in a noticeable reduction of the mean limb intensity between $60^{\circ}$ and $120^{\circ}$ (Fig. 1f). The coronal hole and its associated mean intensity reduction are also observed at $304 \AA$ (Figs. $1 \mathrm{~b}$ and e). At $17 \mathrm{GHz}$ we observe brights patches inside the EUV coronal hole area (Figs. 1a and d) in agreement with previous observations (Gopalswamy et al. 1999; Maksimov et al. 2006).

ii) The north pole also presents a dark area in the delimited region at $171 \AA$ (Fig. 1c); however, it is a filament instead of a coronal hole, as can be seen in the $17 \mathrm{GHz}$ map (Fig. 1a). In this case, intensities at $17 \mathrm{GHz}$ drop to values lower than the quiet Sun level (Fig. 1d). In the EUV images, the reduction in intensity is more prominent at $171 \AA$ (Fig. 1f) than at $304 \AA$, whose emission remains similar to the surrounding regions (Fig. 1e).

\section{The quiet Sun}

The $17 \mathrm{GHz}$ quiet Sun was determined for each individual map using a histogram (bin $=100)$, in which the most common temperature was identified. Then we performed a weighed mean including two values immediately before the histogram peak and two values immediately after it. The result was assumed as the quiet Sun. Since the NoRH are normalized to $10^{4} \mathrm{~K}$, the quiet Sun oscillates around it.

As reported by Boerner et al. (2014), the AIA responses have degraded over the years, most notably in the $304 \AA$ channel. Using the SolarSoft routine aia_get_response.pro, one can see the

\footnotetext{
2 http://solar.nro.nao.ac.jp/norh/images/daily/
} 

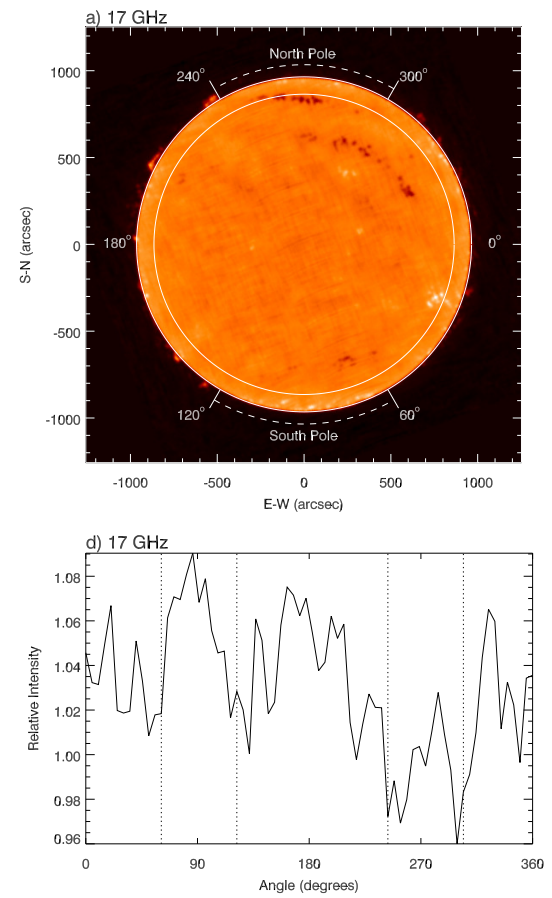
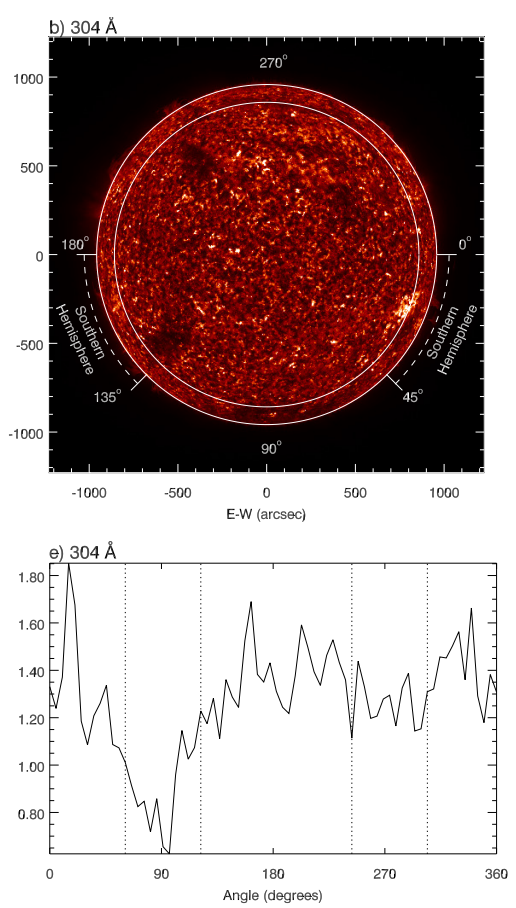
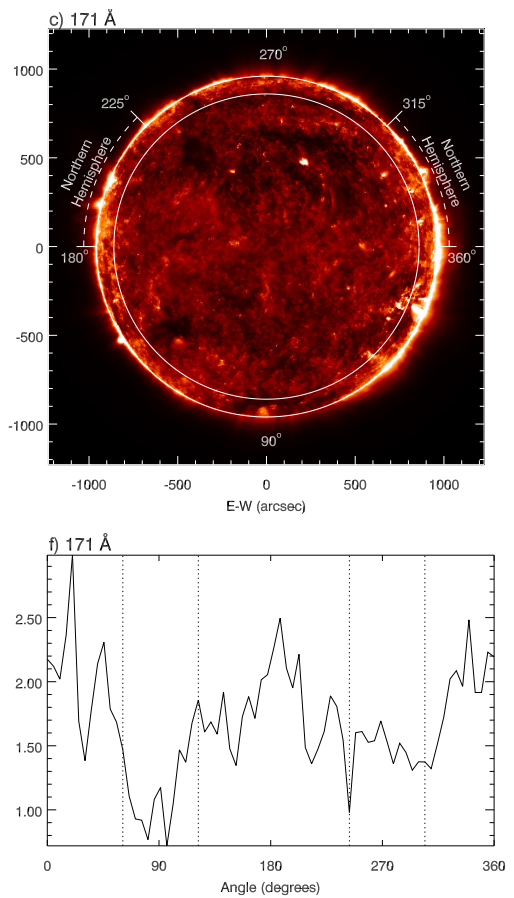

Fig. 1. Solar images observed at a) $17 \mathrm{GHz}$; b) $304 \AA$; and c) $171 \AA$. The concentric circles delimited the analyzed regions and the dashed arcs demarcated the polar regions a), the equatorial southern b) and northern c) hemispheres, respectively. The mean relative intensities $\left(I_{R}\right)$ resulting from the analyses for the three wavelengths are shown in the bottom panels $(\mathbf{d}), \mathbf{e})$ and $\mathbf{f}$ )). The south and north poles were delimited in the ranges $60-120^{\circ}$ and $240-300^{\circ}$, respectively (dotted lines in the panels).

degradation of the AIA responses over time with respect to the EUV Variability Experiment (EVE) on board the SDO. At the end of 2015, the responses of the $171 \AA$ and $304 \AA$ channels decreased to $\sim 86 \%$ and $\sim 20 \%$, respectively, of the initial values as of May 2010. We corrected the AIA $171 \AA$ and $304 \AA$ images for the time-dependent instrument degradation and used them to calculate the quiet Sun intensity in DN with the same procedure that was used to obtain the $17 \mathrm{GHz}$ quiet Sun brightness temperature from the NoRH images. The bin sizes were set to $\sim 1 \%$ of the quiet Sun DN at the beginning of SDO observations, namely, 4 and 1 at $171 \AA$ and $304 \AA$, respectively.

Figure 2 shows the quiet Sun intensity derived in the above procedure. At $171 \AA$, the quiet Sun presents an overall decrease over the years (black dots). The quiet Sun at $304 \AA$ (in gray) stayed almost flat until the latter half of 2014, when it started to decrease from $\sim 100 \mathrm{DN}$ to $\sim 50 \mathrm{DN}$ at the end 2015 . The decrease of the quiet Sun in $304 \AA$ images since 2014 could represent true solar variations. However, it could also be due to residual calibration issues, as the last calibration of the AIA responses with the EVE took place in 2014, which is called version 6 . The EVE calibration rocket scheduled for launch in 2016 will help us identify the origin of the downturn of the $304 \AA$ quiet Sun that started in 2014. We note that the synoptic results as presented in the next section and Fig. 3 are not strongly affected by the apparent decrease of the quiet Sun intensity at $304 \AA$.

\section{Results}

In the studied period (2010-2015), around 2000 images were analyzed for each wavelength, after manually checking and discarding images with problems $(<2 \%)$. The results at $17 \mathrm{GHz}$ show that $I_{R}$ in the $5^{\circ}$ cells varies between 0.70 and 29.02; the lower values could be addressed as the presence of dark filaments in the delimited region, and the higher values are due to

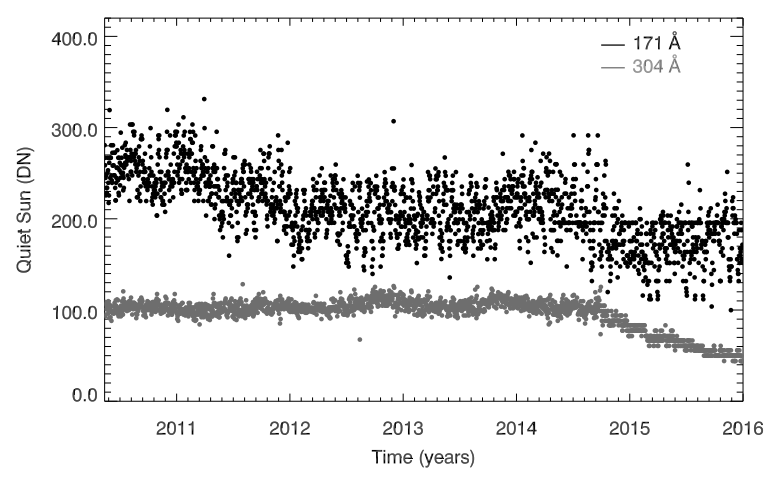

Fig. 2. AIA quiet Sun variation at $171 \AA$ (black) and $304 \AA$ (gray). The $\mathrm{DN}$ values are corrected for the time-dependent instrument responses.

the presence of extreme active regions, which can reach brightness temperatures above $5 \times 10^{5} \mathrm{~K}$, i.e. 50 times greater than the quiet Sun values (Selhorst et al. 2014, and references therein). We attribute the stronger emission from active regions to the gyro-resonance contribution that becomes important in the presence of strong magnetic fields $(\sim 2000 \mathrm{G})$ in the transition region or at coronal heights (Shibasaki et al. 2011). Nevertheless, these extreme active regions are rarely observed at the limb in the analyzed period: only 24 days present relative intensities $I_{R} \geqslant 5$ and 6 days have $I_{R} \geqslant 10$.

In EUV filter images, the presence of coronal holes results in a stronger decrease of limb emission with 304 and $171 \AA$ reaching $I_{R}$ minimum values of 0.57 and 0.49 , respectively. On the other hand, the active regions at the limb increase $I_{R}$ at a lesser pace than in radio with a maximum around 18.11 at $304 \AA$ and 26.42 at $171 \AA$, which likely reflects the single free-free emission mechanism that is not affected by strong magnetic field in active regions. 

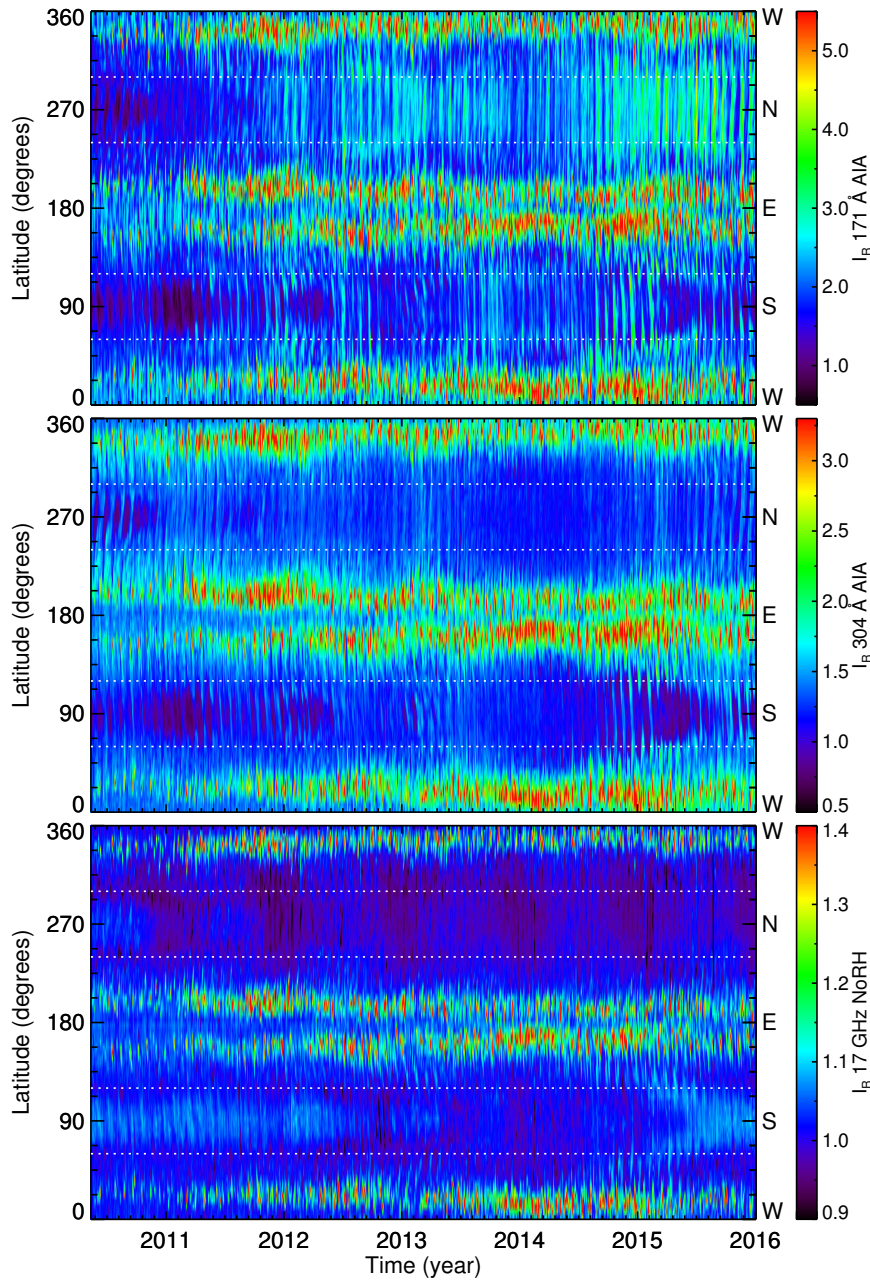

Fig. 3. Synoptic limb charts obtained for $171 \AA$ A (top panel), $304 \AA$ (middle panel) and $17 \mathrm{GHz}$ (bottom panel). Running means of 3 days were applied in the synoptic charts of each wavelength. The minimum values of the color scales for the $I_{R}$ are 0.5 at the EUV (171 and $304 \AA$ ) lines and 0.70 at radio $(17 \mathrm{GHz})$, while the maximum values are $5.50,3.30$ and 1.40 at $171 \AA, 304 \AA$ and $17 \mathrm{GHz}$, respectively. The dotted lines delimit the south $\left(60^{\circ}\right.$ and $\left.120^{\circ}\right)$ and north $\left(240^{\circ}\right.$ and $\left.300^{\circ}\right)$ poles.

The daily $I_{R}$ angular distributions are organized in synoptic limb charts, in which the angular position is represented in the ordinates, and the time in the abscissas; intensities are colorcoded. Since our main objective is to study the behavior of the polar regions as a whole, the $I_{R}$ values in Fig. 3 are artificially saturated at 5.50, 3.30, and 1.40 at $171 \AA, 304 \AA$, and $17 \mathrm{GHz}$, respectively, to enhance the visualization of the tenuous variations at the polar regions. We now discuss the results in terms of the equatorial and polar regions separately.

\subsection{Equatorial emission}

We calculated the monthly mean emission in the northern and southern hemispheres to quantify the intensity increase in the equatorial region observed in Fig. 3 and verify a possible northsouth asymmetry in the solar activity. The monthly mean equatorial emission in the southern hemisphere is here defined as the monthly average of intensities in the ranges 0 to $45^{\circ}$ and 135 and $180^{\circ}$ (see Fig. 1b). In the northern hemisphere, it is defined as the monthly average between $180-225^{\circ}$ and $315-360^{\circ}$ (see Fig. 1c). The results are shown in Fig. 4. For comparison with
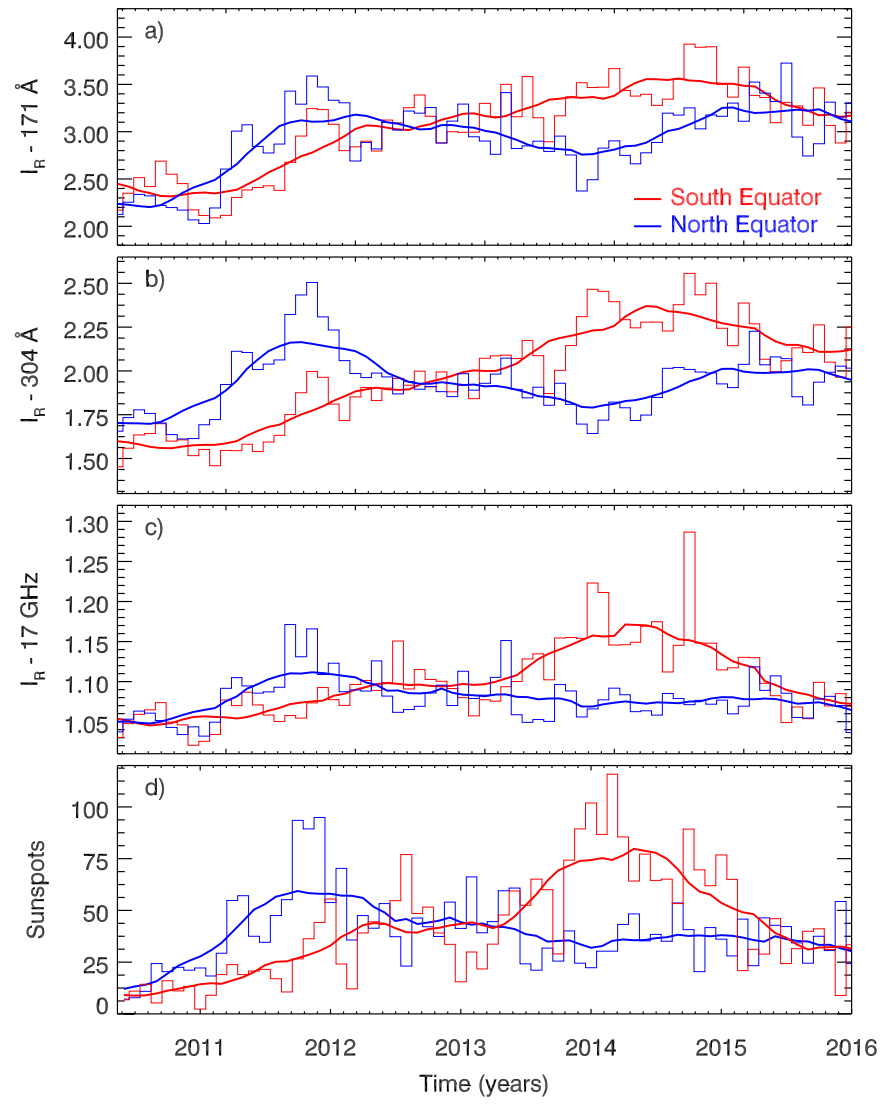

Fig. 4. Monthly mean equatorial $I_{R}$ at a) $171 \AA$; b) $304 \AA$ and c) $17 \mathrm{GHz}$. The red and blue curves are the mean equatorial $I_{R}$ in the south and north hemispheres, respectively. For comparison, the monthly sunspot number for each hemisphere are also shown d). Yearly running means, i.e., every 12 points, were applied (thick lines).

the solar cycle, the bottom panel shows the monthly sunspot number (SSN) for each hemisphere ${ }^{3}$. The southern and northern hemispheres are shown in red an blue, respectively, and yearly running averages (12 points) were applied to all the values (thick lines).

The equatorial results were are consistent with the hemispheric sunspot number. The three wavelengths have similar mean equatorial intensity $\left(\left\langle I_{R}\right\rangle\right)$ variations, which is evident from the significant correlation coefficients between the radio and the EUV for the monthly averages (Table 1). Following the sunspot number, the synoptic charts showed a northern dominance until 2012 and the increase in the southern activity at the end of 2013. The differences between the equatorial hemispheric emission were more evident at the chromospheric level, at $304 \AA$ and $17 \mathrm{GHz}$ (Figs. 4b and c), which resulted in high correlation indexes, 0.79 and 0.80 , for the southern and northern hemispheres, respectively.

The hemispheric differences at $171 \AA$ (Fig. 4a ) are smaller than those observed in the chromosphere, however, the behavior is still the same. Moreover, good correlations were found in the comparison with the $17 \mathrm{GHz}$ monthly values, namely 0.73 for the south and 0.62 for the north.

3 Obtained at http://www.sidc.be/silso/datafiles 
Table 1. Correlation coefficients between radio and EUV results.

\begin{tabular}{|c|c|c|c|}
\hline & & \multicolumn{2}{|c|}{$17 \mathrm{GHz}$ correl. coefficients } \\
\hline & & South & North \\
\hline \multirow[t]{2}{*}{ Equator } & vs. $171 \AA$ & 0.73 & 0.62 \\
\hline & vs. $304 \AA$ & 0.79 & 0.80 \\
\hline \multirow[t]{2}{*}{ Pole } & vs. $171 \AA$ & -0.39 & -0.36 \\
\hline & vs. $304 \AA$ & -0.28 & -0.04 \\
\hline
\end{tabular}

Notes. The correlation coefficients between the monthly average of the radio $17 \mathrm{GHz}$ emission and the EUV emission at 171 and $304 \AA$. The analises were separated in the hemispheric mean polar and equatorial emission.

\subsection{Polar emission}

While in the equatorial analysis the EUV and radio emission showed a good agreement, at the poles the scenario changes completely and the differences between EUV and radio are clear. At $171 \AA$ the presence of coronal holes, observed as dark regions at the poles (Fig. 3 top), are reflected in the synoptic map as a decrease in the mean intensity. The dark regions remain stable for months and are present in high numbers at the south pole. At the north pole, the last large coronal hole was observed at the end of 2010, but coronal holes can be observed in the southern hemisphere until the first half of 2012 and after 2015. Without the coronal holes both poles present an increase at $171 \AA$. The coronal holes observed at $171 \AA$ were also identified in the $304 \AA$ synoptic chart (Fig. 3 middle). On the other hand, at $17 \mathrm{GHz}$, instead of coronal holes the poles present a prominent intensity increase (Fig. 3 bottom) until 2012 and after 2015. In the absence of the coronal holes (around 2013), $I_{R}$ decreases.

In order to compare the temporal variation of the polar regions, the mean $\left\langle I_{R}\right\rangle$ of the south $\left(60^{\circ}\right.$ and $\left.120^{\circ}\right)$ and north $\left(240^{\circ}\right.$ and $\left.300^{\circ}\right)$ poles were calculated for each month. Results are shown in Fig. 5, and present the following characteristics:

- $171 \AA$ : the mean $\left\langle I_{R}\right\rangle$ increases at both poles (Fig. 5a) with the north (blue curve) more intense than the south pole (red curve);

- $304 \AA$ : the mean $\left\langle I_{R}\right\rangle$ is almost constant at the north pole with a small reduction in the beginning of 2014 (Fig. 5b, blue curves). In the south the mean $\left\langle I_{R}\right\rangle$ started to increase with the disappearance of coronal holes and reached a maximum in 2013. Then $\left\langle I_{R}\right\rangle$ decreased to a minimum at the end of 2014 and, even with the presence of a coronal hole, it has started to increase again (Fig. 5b, red curves);

- $17 \mathrm{GHz}$ : contrarily to the EUV, the south pole presented greater mean $\left\langle I_{R}\right\rangle$ values during the analyzed period. The north pole showed only small variations in the mean $\left\langle I_{R}\right\rangle$ during the analyzed period (Fig. 5c, blue curves). The south pole varied in the opposite way to the sunspot number, i.e., the mean $\left\langle I_{R}\right\rangle$ decreased in the period in which the sunspot number reached the maximum (red curves in Figs. $5 \mathrm{c}$ and $\mathrm{d}$ ).

The correlation between the poles in radio and EUV resulted in lower values than those obtained at the equator. In the south the indexes comparing $17 \mathrm{GHz}$ with 171 and $304 \AA$ i were -0.39 and -0.36 , respectively. These negative values can be attributed to the presence of coronal holes, which are dark at EUV, but presented a bright counterpart at $17 \mathrm{GHz}$. At the north pole, the coronal holes were less frequently observed, which resulted in a correlation index of -0.28 , between $17 \mathrm{GHz}$ and $171 \AA$ and a

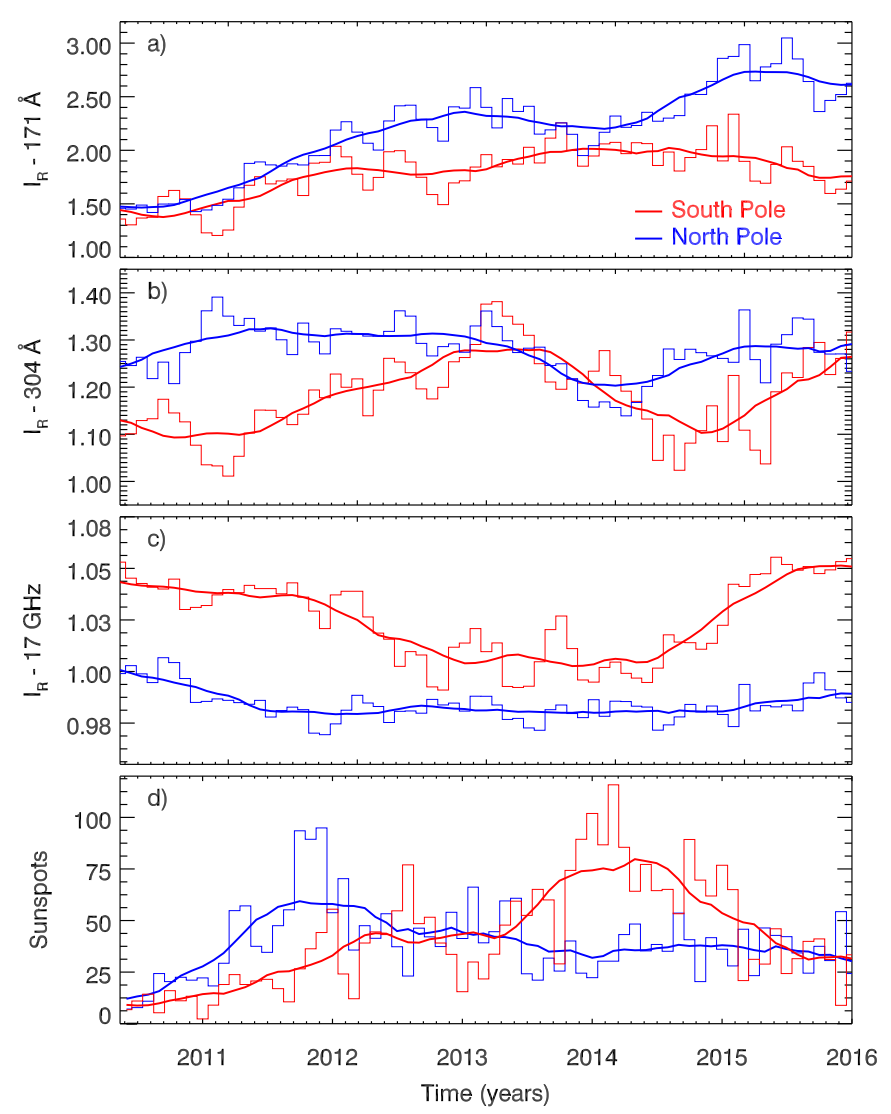

Fig. 5. Monthly mean polar $I_{R}$ variations at a) $171 \AA$, b) $304 \AA$ and c) $17 \mathrm{GHz}$. For comparison the monthly sunspot numbers for each hemisphere were plotted in panel d). The daily south (red curves) and north (blue curves) means $I_{R}$ were calculated in the regions $60-120^{\circ}$ and $240-300^{\circ}$, respectively. Yearly running means (12 points) were applied to the values (thick lines).

index close to zero $(-0.04)$ in the comparison between $17 \mathrm{GHz}$ and $304 \AA$.

\section{Discussion and conclusions}

We analyzed the polar and equatorial emission at $17 \mathrm{GHz}$, $171 \AA$ and $304 \AA$ through synoptic limb charts constructed from NoRH and AIA maps. The synoptic charts cover the period of 2010-2015 show increasing solar activity in the equatorial region and a reduction in the radio polar brightening during the period of maximum activity.

\subsection{Equatorial region}

At the equatorial region, the results for the three wavelengths clearly show the hemispheric asymmetry in the solar activity (see for example: Hathaway 2010). The dominance of the northern hemisphere coincided with the first SSN peak, whereas the second SSN peak occurs simultaneously with increase in the activity at the south (see Fig. 4). There is an interval between the peaks (around the beginning of 2013), which indicates an inversion in the asymmetry of the hemispheric activity. Moreover, the comparison between the hemispheres shows that the peak of activity in the south is more intense for all three wavelengths.

The stronger southern activity started in the first semester of 2013 and is coincident with the complete polar field reversion of the north pole (Mordvinov et al. 2015). Since solar 
cycle 20, polar field reversals first occurred in the north (Svalgaard \& Kamide 2013), all those cycles showed a more intense first peak. This trend has clearly changed in the present cycle, being the more intense the second peak.

The monthly equatorial radio $\left\langle I_{R}\right\rangle$ in both hemispheres presented an overall positive correlation with the EUV (see Table 1). The trend has clearly changed in the present cycle, in which the second peak is more intense. A stronger activity in the southern hemisphere was more noticeable at $17 \mathrm{GHz}$ and $304 \AA$, which both formed in the lower atmosphere.

\subsection{Polar regions}

In the polar regions, the presence of stable and long-lived coronal holes is evident at both EUV wavelengths; this is identified by the darker patches in Fig. 3. We also note an asymmetry of their hemispheric activity, in which the darker patches at the north pole started to disappear earlier than at the south pole, in agreement with the results obtained by Karna et al. (2014). Remarkably, the south pole showed coronal holes in the beginning of 2015 , whereas no coronal holes were observed at the north pole in the same period.

The $17 \mathrm{GHz}$ radio emission at the south pole clearly decreases while the SSN increases, and vice versa (Fig. 5); on the other hand, the radio emission at the north pole is almost constant during the studied period. For the EUV bands, $\left\langle I_{R}\right\rangle$ at the north pole is more intense than the $\left\langle I_{R}\right\rangle$ at the south throughout the entire analyzed period. At $17 \mathrm{GHz}$, the south pole dominated in the period (cf. Selhorst et al. 2011; Gopalswamy et al. 2012; Nitta et al. 2014).

This work strengthens the association between coronal holes and the $17 \mathrm{GHz}$ polar brightenings as it is evident in the synoptic limb charts shown in Fig. 3, in agreement with previous case study works (Gopalswamy et al. 1999; Maksimov et al. 2006). The enhancement of the radio brightness in coronal holes is explained by the presence of bright patches closely associated with the presence of increased unipolar magnetic regions underlying the coronal holes (Gopalswamy et al. 1999; Brajša et al. 2007; Selhorst et al. 2010). However, the physical mechanisms that link the radio brightenings and coronal holes are not yet fully understood. Observations of these brightenings with better spatial resolution and also at different radio wavelengths, such as solar observations with the Atacama Large Millimetric/Submillimetric Array (ALMA) (Wedemeyer et al. 2016) might be fundamental to investigate this association.
Acknowledgements. The authors would like to thank the referee for valuable comments and suggestions that helped to improve the manuscript. We also thank Peter Levens for language corrections. We would like to thank the Nobeyama Radioheliograph, which is operated by the NAOJ/Nobeyama Solar Radio Observatory. A.J.O.S. acknowledge the scholarship form CAPES. C.L.S. acknowledge financial support from the São Paulo Research Foundation (FAPESP), grant number 2014/10489-0.

\section{References}

Andretta, V., \& Jones, H. P. 1997, ApJ, 489, 375

Boerner, P., Edwards, C., Lemen, J., et al. 2012, Sol. Phys., 275, 41

Boerner, P. F., Testa, P., Warren, H., Weber, M. A., \& Schrijver, C. J. 2014, Sol. Phys., 289, 2377

Brajša, R., Benz, A. O., Temmer, M., et al. 2007, Sol. Phys., 245, 167

Del Zanna, G., \& Andretta, V. 2011, A\&A, 528, A139

Freeland, S. L., \& Handy, B. N. 1998, Sol. Phys., 182, 497

Glackin, D. L., Linsky, J. L., Mango, S. A., \& Bohlin, J. D. 1978, ApJ, 222, 707

Gopalswamy, N., Shibasaki, K., Thompson, B. J., Gurman, J., \& DeForest, C. 1999, J. Geophys. Res., 104, 9767

Gopalswamy, N., Yashiro, S., Mäkelä, P., et al. 2012, ApJ, 750, L42

Hathaway, D. H. 2010, Liv. Rev. Sol. Phys., 7, 1

Hirayama, T. 1971, Sol. Phys., 17, 50

Jordan, C. 1975, MNRAS, 170, 429

Karna, N., Hess Webber, S. A., \& Pesnell, W. D. 2014, Sol. Phys., 289, 3381

Koshiishi, H. 2003, A\&A, 412, 893

Lemen, J. R., Title, A. M., Akin, D. J., et al. 2012, Sol. Phys., 275, 17

Maksimov, V. P., Prosovetsky, D. V., Grechnev, V. V., Krissinel, B. B., \& Shibasaki, K. 2006, PASJ, 58, 1

Mordvinov, A. V., Grigoryev, V. M., \& Erofeev, D. V. 2015, Adv. Space Res., 55,2739

Nakajima, H., Nishio, M., Enome, S., et al. 1994, IEEE Proc., 82, 705

Nindos, A., Kundu, M. R., White, S. M., et al. 1999, ApJ, 527, 415

Nitta, N. V., Sun, X., Hoeksema, J. T., \& DeRosa, M. L. 2014, ApJ, 780, L23

Pauluhn, A., \& Solanki, S. K. 2003, A\&A, 407, 359

Riehokainen, A., Urpo, S., Valtaoja, E., et al. 2001, A\&A, 366, 676

Selhorst, C. L., Silva, A. V. R., \& Costa, J. E. R. 2005, A\&A, 433, 365

Selhorst, C. L., Giménez de Castro, C. G., Varela Saraiva, A. C., \& Costa, J. E. R. 2010, A\&A, 509, A51

Selhorst, C. L., Giménez de Castro, C. G., Válio, A., Costa, J. E. R., \& Shibasaki, K. 2011, ApJ, 734, 64

Selhorst, C. L., Costa, J. E. R., Giménez de Castro, C. G., et al. 2014, ApJ, 790, 134

Shibasaki, K., Alissandrakis, C. E., \& Pohjolainen, S. 2011, Sol. Phys., 273, 309

Steer, D. G., Dewdney, P. E., \& Ito, M. R. 1984, A\&A, 137, 159

Svalgaard, L., \& Kamide, Y. 2013, ApJ, 763, 23

Tsuneta, S., Ichimoto, K., Katsukawa, Y., et al. 2008, ApJ, 688, 1374

Wedemeyer, S., Bastian, T., Brajša, R., et al. 2016, Space Sci. Rev., 200, 1

Worden, J., Woods, T. N., Neupert, W. M., \& Delaboudinière, J.-P. 1999, ApJ, 511,965

Zirin, H. 1975, ApJ, 199, L63 